\title{
Meningeal Lymphangiogenesis and Enhanced Glymphatic Activity in Mice with Chronically Implanted EEG Electrodes
}

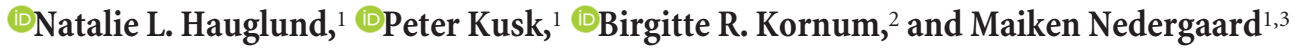 \\ ${ }^{1}$ Center for Translational Neuromedicine, Faculty of Health and Medical Sciences, University of Copenhagen, 2200 Copenhagen, Denmark, ${ }^{2}$ Department of \\ Neuroscience, Faculty of Health and Medical Sciences, University of Copenhagen, 2200 Copenhagen, Denmark, and ${ }^{3}$ Center for Translational \\ Neuromedicine, University of Rochester Medical Center, Rochester, New York 14642
}

Chronic electroencephalography (EEG) is a widely used tool for monitoring cortical electrical activity in experimental animals. Although chronic implants allow for high-quality, long-term recordings in preclinical studies, the electrodes are foreign objects and might therefore be expected to induce a local inflammatory response. We here analyzed the effects of chronic cranial electrode implantation on glymphatic fluid transport and in provoking structural changes in the meninges and cerebral cortex of male and female mice. Immunohistochemical analysis of brain tissue and dura revealed reactive gliosis in the cortex underlying the electrodes and extensive meningeal lymphangiogenesis in the surrounding dura. Meningeal lymphangiogenesis was also evident in mice prepared with the commonly used chronic cranial window. Glymphatic influx of a CSF tracer was significantly enhanced at $30 \mathrm{~d}$ postsurgery in both awake and ketaminexylazine anesthetized mice with electrodes, supporting the concept that glymphatic influx and intracranial lymphatic drainage are interconnected. Altogether, the experimental results provide clear evidence that chronic implantation of EEG electrodes is associated with significant changes in the brain's fluid transport system. Future studies involving EEG recordings and chronic cranial windows must consider the physiological consequences of cranial implants, which include glial scarring, meningeal lymphangiogenesis, and increased glymphatic activity.

Key words: astrogliosis; CSF; dura; glial scarring; lymphatic; meninges

\section{Significance Statement}

This study shows that implantation of extradural electrodes provokes meningeal lymphangiogenesis, enhanced glymphatic influx of CSF, and reactive gliosis. The analysis based on CSF tracer injection in combination with immunohistochemistry showed that chronically implanted electroencephalography electrodes were surrounded by lymphatic sprouts originating from lymphatic vasculature along the dural sinuses and the middle meningeal artery. Likewise, chronic cranial windows provoked lymphatic sprouting. Tracer influx assessed in coronal slices was increased in agreement with previous reports identifying a close association between glymphatic activity and the meningeal lymphatic vasculature. Lymphangiogenesis in the meninges and altered glymphatic fluid transport after electrode implantation have not previously been described and adds new insights to the foreign body response of the CNS.

\section{Introduction}

A broad range of methodologies in contemporary neuroscience research require chronic cranial implantations. For example, the implantation of cranial electrodes for long-term

\footnotetext{
Received Sept. 14, 2019; revised Dec. 27, 2019; accepted Jan. 22, 2020.

Author contributions: N.L.H., B.R.K., and M.N. designed research; N.L.H. and P.K. performed research; N.L.H. analyzed data; N.L.H. and M.N. wrote the paper.

This work was supported by the Novo Nordisk Foundation, the Lundbeck Foundation, and the Adelson Medical Research Foundation. We thank Dan Xue for expert graphical support and Paul Cumming for comments on the paper.

The authors declare no competing financial interests.

Correspondence should be addressed to Maiken Nedergaard at Maiken_Nedergaard@URMC.Rochester.edu.

https://doi.org/10.1523/JNEUROSCI.2223-19.2020

Copyright $\odot 2020$ the authors
}

electroencephalographic (EEG) recordings involves insertion of cranial screws or positioning of an electrode array resting on top of the dura. Earlier studies have shown that cortical astrocytes and microglia exhibit reactive changes in response to such procedures (Benveniste and Diemer, 1987; Turner et al., 1999; Szarowski et al., 2003; Schendel et al., 2014). However, those studies were performed before the discovery of meningeal lymphatic vessels (Aspelund et al., 2015; Louveau et al., 2015; Absinta et al., 2017; Visanji et al., 2018), and it therefore remains to be established how the lymphatic vasculature and likewise immune cells resident in the dura may respond to cranial implants.

In the setting of injury or inflammation within the CNS, astrocytes become reactive and upregulate GFAP (Landis, 1994). 
Microglia cells are also activated, leading to proliferation and morphological changes (Saijo and Glass, 2011), and furthermore the perturbed blood-brain barrier will often display transient leakage (Sawyer and Kyriakides, 2013). In peripheral tissues, injury or foreign objects elicit lymphangiogenesis (Ji, 2007; Tammela and Alitalo, 2010), and a similar response might be expected to occur in meningeal lymphatic vessels within the CNS. However, a recent examination of a mouse model of multiple sclerosis (the EAE model) noted localized sprouting of lymphatic vessels near the cribriform plate, whereas lymphatic vessels in the meninges of brain or spinal cord did not exhibit any such reactive responses (Louveau et al., 2018; Hsu et al., 2019).

Unlike other organs, the brain parenchyma is itself devoid of lymphatic vessels. Instead, metabolic waste products are removed from the brain extracellular space by flow of CSF and ultimately egress via the glymphatic pathway along perivascular channels (Iliff et al., 2012). CSF is produced in the choroid plexus of the ventricles and disperses along the subarachnoid space surrounding the brain. From there it enters the brain parenchyma via the peri-arterial spaces that are permissive to CSF inflow when the brain is in a functional state of high slow-wave power, as occurs during non-REM (nREM) sleep or under select anesthesia regimens (Iliff et al., 2012; Xie et al., 2013; Benveniste et al., 2017; Fultz et al., 2019; Hablitz et al., 2019). CSF interchanges with interstitial fluid that contains waste metabolites produced by brain cells, and then disperses toward the peri-venous exit paths to leave the CNS via drainage into the lymphatic network. In this way, the brain can export toxic substances, most notably soluble amyloid $\beta$ (Iliff et al., 2012). It would follow that inflammatory responses resulting from EEG electrode implantation (Turner et al., 1999; Szarowski et al., 2003; Schendel et al., 2014) might cause long-term changes in glymphatic function, but this remains to be determined.

We here systematically assessed the effect of electrode implantation on both the glymphatic and the meningeal lymphatic systems. The analysis confirmed that electrode implantation results in a chronic local reactive response from astrocytes and microglial cells, occurring concurrently with a major structural remodeling of the dura and its lymphatic vessels and an increase in glymphatic influx.

\section{Materials and Methods}

Animals. Studies were approved by the local research ethics committee and conducted in accordance with the Danish Animal Experiments Inspectorate. All animals were housed in a $12 \mathrm{~h}$ light/dark cycle with ad libitum access to food and water, and in agreement with existing guidelines. For sleep phenotyping, glymphatic analysis, and immunohistochemistry, we used $\sim 12$-week-old male C57BL/6JRj mice (Janvier) weighing 26-31 g at the time of surgery. For lymphatic visualization, 10to 40 -week-old male and female Prox-1 mice were used (the line was kindly shared by Dr. Kari Alitalo; University of Helsinki) on a C57BL/ 6JRj background, weighing 24-31 g at the time of surgery. Prox1 EGFP mice express enhanced green fluorescent protein (EGFP) under the Prox-1 promoter, which allowed for direct visualization of lymphatic vasculature (Choi et al., 2011; Antila et al., 2017). Prox-1 EGFP mice were used in this study because the fluorescent signal in transgene Prox-1 EGFP mice has been reported by several groups to colocalize with other known lymphatic markers and reliably display lymphatic vasculature (Choi et al., 2011; Aspelund et al., 2015; Cai et al., 2019). Care was taken to minimize the number of mice used.

EEG electrode implantation. All surgical procedures were performed by practitioners with extensive experience in rodent surgery. Mice were anesthetized with isoflurane and placed on a heating pad, and surgical tools were cleaned with ethanol just before use. Carprofen $(5 \mathrm{mg} / \mathrm{kg})$ and buprenorphine $(0.05 \mathrm{mg} / \mathrm{kg})$ were injected subcutaneously, and lido- caine $(1 \mathrm{mg} / \mathrm{ml})$ was injected locally at the surgical site. After confirming lack of pain reflexes, the neck region was shaved and cleaned with ethanol and chlorhexidine solution, and the skull was exposed by making a $\sim 1.5$ $\mathrm{cm}$ incision in the scalp between the ears. The exposed skull was cleaned and scratched with a pair of tweezers to improve later binding between skull and dental cement. Four holes ( $0.8 \mathrm{~mm}$ in diameter) were drilled in the skull with a handheld electrical drill (Tech2000, RAM Microtorque; $45,000 \mathrm{rpm}$ ), taking great care not to damage the underlying dura or brain tissue. To minimize heat production, drilling was performed in 2-3 s intervals with 1-2 min breaks in between, thus allowing the tissue to cool down (Silasi et al., 2013; Goldey et al., 2014; Goss-Varley et al., 2018). The four burr holes were positioned bilaterally above the parietal and frontal cortex by using bregma, lambda, and the sagittal suture as visual reference points. One pair of holes was drilled over the right cerebral hemisphere ( $1 \mathrm{~mm}$ lateral to the midline, $1 \mathrm{~mm}$ anterior to bregma; $1 \mathrm{~mm}$ lateral to the midline, $1 \mathrm{~mm}$ anterior to lambda), and another pair was drilled above the left hemisphere (5-6 mm lateral to the midline and $1 \mathrm{~mm}$ anterior to bregma or lambda). A pair of $0.8 \mathrm{~mm}$ stainless steel anchor screws was screwed into burr holes on the left hemisphere, and a pair of $0.8 \mathrm{~mm}$ low impedance stainless steel screws was placed in the remaining burr holes on the right hemisphere (NeuroTek). A silver wire (W3 Wires International) inserted into the trapezius muscle served as an electromyogram (EMG) electrode. Dental glue (Panavia SA Cement Plus, Kuraray) and dental cement (ProBase) were applied to cover the screw heads. Carprofen $(5 \mathrm{mg} / \mathrm{kg}$ ) was injected subcutaneously following the surgery, and again at 24 and $48 \mathrm{~h}$ post-surgery. Control mice were non-operated littermates.

EEG recordings and sleep scoring. Fourteen days after surgery, mice were briefly anesthetized with isoflurane and cables were connected to the EEG and EMG electrodes. Cables were connected to a commutator (Plastics One) and the mice were allowed to habituate to the recording chamber (ViewPoint Behavior Technology) for $3 \mathrm{~d}$. Signals were amplified (National Instruments), and filtered (EEG signal: high-pass at $1 \mathrm{~Hz}$ and low-pass at $100 \mathrm{~Hz}$; EMG signal: high-pass at $10 \mathrm{~Hz}$ and low-pass at $100 \mathrm{~Hz}$ ). A notch filter of $50 \mathrm{~Hz}$ was used to reduce power line noise. Signals were digitized using a NI Usb 6343 card (National Instruments) and sampled at a sampling rate of $512 \mathrm{~Hz}$. Video was continuously recorded using an infrared video camera (Flir Systems) and used later to aid in the scoring of vigilance states. Hypnograms were created by visual inspection from EEG traces for five second epochs. Vigilance states were defined as wake (muscle tonus and a high-frequency, low-amplitude EEG), nREM sleep (no muscle tonus and low-frequency, high-amplitude EEG,), and REM sleep (no muscle tonus and high-frequency, lowamplitude EEG). Analysis of hypnograms was done using SleepScore software (ViewPoint Behavior Technology; Libourel et al., 2015; Roman et al., 2018; Rosier et al., 2018).

Immobility assay. The immobility assay (Pack et al., 2007) was performed in the same cages as the EEG recordings with ad libitum access to food and water. An infrared video camera (Flir Systems) was used to monitor the mice over a $24 \mathrm{~h}$ period and immobility was thereafter detected automatically using the mobility parameter in EthoVision XT (Noldus).

Cranial window. For experiments testing the effect of cranial window implantation, a separate group of mice was used. These mice were anesthetized with isoflurane and received preoperative buprenorphine $(0.05$ $\mathrm{mg} / \mathrm{kg}$, s.c.) and lidocaine ( $1 \mathrm{mg} / \mathrm{ml}$, s.c., at surgery site). After confirming lack of pain reflexes, skin was removed from the scalp and a custom made headplate was positioned to allow head fixation on a Narishige MAG-1 head plate holder device. A dental drill with a 009 size drill bit attached was used to make a 3-mm-diameter cranial window over the right visual cortex under perfusion with HEPES buffered aCSF. A precut 3-mm-diameter round coverslip (ThermoFisher) was placed on top of the dura and sealed in place with a gel cyanoacrylate glue. Carprofen $(5 \mathrm{mg} / \mathrm{kg})$ was injected subcutaneously following the surgery and again at 24 and $48 \mathrm{~h}$ post-surgery. Macroscopic cortical imaging was performed with a fluorescence stereomicroscope (Leica M205 FA) with a Hamamatsu ORCA-Flash4.0 V2 Digital CMOS camera using LAS X Leica software. 
Cisterna magna injections. Mice were anesthetized with isoflurane (awake injection groups) or ketamine-xylazine (ketamine-xylazine injection groups) and injected preoperatively with lidocaine $(1 \mathrm{mg} / \mathrm{ml}$, s.c.) locally at the surgical site. Mice in the awake groups were further injected preoperatively with carprofen $(5 \mathrm{mg} / \mathrm{kg})$ and buprenorphine $(0.05 \mathrm{mg} /$ $\mathrm{kg})$. The atlanto-occipital membrane was exposed and the tip of a 30 gauge needle was placed in the cisterna magna and fixed with glue and dental cement (ProBase). After placement of the needle, mice in the awake injection groups were allowed to recover for $2 \mathrm{~d}$ before tracer injection, while mice in the ketamine-xylazine groups had tracer injected immediately following surgery. Twelve microliters of $2 \%$ fluoresceinconjugated dextran (3 kDa; ThermoFisher, D3305) in artificial CSF was slowly infused into the cisterna magna over a period of $12 \mathrm{~min}$. Thirty minutes after the end of the tracer infusion, mice were killed by cervical dislocation and the brain was immediately dissected out and fixed overnight in $4 \%$ PFA at $4^{\circ} \mathrm{C}$. Brains were then cut in $100-\mu \mathrm{m}$-thick coronal slices on a vibratome (VT1200S, Leica Biosystems), and seven brain sections spaced evenly across the anterior-posterior axis were stained with 4',6-diamidin-2-phenylindol (DAPI; ThermoFisher) and mounted with PROLONG Anti-Fade Gold (Life Technologies). Note that glymphatic tracer distribution in the awake and the ketamine-xylazine groups cannot be directly compared since different surgical procedures were used and the experiments were performed 7 months apart. As a consequence, the CSF tracer used in the two sets of experiments was from different batches and the microscope settings were not identical.

Tracer analysis. Brain sections were imaged on a Nikon Eclipse NI-E epifluorescence microscope using NIS Elements software. Multichannel whole-slice montages were generated at $4 \times$ magnification with emission filters for DAPI and fluorescein isothiocyanate (FITC) with constant exposure levels throughout the study. Mean pixel intensities were calculated for each of the seven coronal brain sections using ImageJ software (NIH). For regional analysis, the second section ( $1 \mathrm{~mm}$ from bregma) was divided into regions (lateral cortex, ventral cortex, medial septum region) and mean pixel intensities were calculated for each region.

Histological preparation. Mice were killed by cervical dislocation and immediately thereafter the brain was removed and immersion-fixed overnight in $4 \%$ paraformaldehyde (PFA) at $4^{\circ} \mathrm{C}$. The following day, brains were cut in $100-\mu \mathrm{m}$-thick coronal slices on a vibratome (VT1200S, Leica Biosystems) and mounted with PROLONG Anti-Fade Gold (Life Technologies). Whole-skull dura preparations were prepared by dissecting and removing the dorsal part of the skull with the dura still attached, and leaving these samples for overnight fixation in $4 \%$ PFA at $4^{\circ} \mathrm{C}$. For histological preparations showing meningeal blood vasculature, mice were deeply anesthetized with ketamine $(100 \mathrm{mg} / \mathrm{kg})$ and xylazine $(10 \mathrm{mg} / \mathrm{kg})$ and perfused transcardially with saline followed by AlexaFluor 594-labeled wheat germ agglutinin (ThermoFisher Scientific), and finally $4 \%$ PFA. Skull and dura were dissected and fixed by immersion fixation overnight in $4 \%$ PFA at $4^{\circ} \mathrm{C}$.

Immunohistochemistry and imaging. Brain sections and skull + dura were washed in PBS and incubated with blocking solution (0.3\% Triton $\mathrm{X}-100,5 \%$ serum, PBS) for $2 \mathrm{~h}$ at room temperature. Samples were then incubated overnight at $4^{\circ} \mathrm{C}$ with primary antibodies (see next section). The following day, samples were washed with PBS and incubated with secondary antibodies for $2 \mathrm{~h}$ at room temperature while protected from light. After incubation, samples were washed with PBS and incubated for 5 min with DAPI (ThermoFisher Scientific) diluted in PBS (1:1000), followed by additional washes in PBS. Finally, brain sections and dura were mounted to glass slides with mounting medium (ProLong Gold Antifade, ThermoFisher Scientific) and coverslipped, while skull-dura preparations were kept in in PBS at $4^{\circ} \mathrm{C}$ until being imaged. Brain slices and dura were imaged on a Nikon Eclipse NI-E epifluorescence microscope using NIS Elements software. Multichannel whole-slice montages were generated at $4 \times$ magnification with emission filters appropriate for DAPI, FITC, and far red. Preparations of dura were imaged on a Nikon Eclipse Ti confocal laser scanning microscope. Magnification images $(10 \times)$ were stitched to whole-slice montages using NIS Elements software with emission filters for DAPI, FITC, and far red. Skull-dura preparations were imaged on a fluorescence stereomicroscope (Leica M205
FA) with a Hamamatsu ORCA-Flash4.0 V2 Digital CMOS camera using LAS X Leica software.

Antibodies. For brain sections, primary antibodies against GFAP (chicken- $\alpha$ GFAP, 1:1000; ThermoFisher Scientific, PA110004), CD68 (rat- $\alpha$ mouse CD68, 1:100; Bio-Rad, MCA1957), and AQP4 (rabbit- $\alpha$ mouse AQP4, 1:200; ThermoFisher Scientific, PA536521) were used. Corresponding secondary antibodies were goat $\alpha$-chicken IgY (1:500; ThermoFisher Scientific), goat $\alpha$-rat IgG (1:500; ThermoFisher Scientific), and goat $\alpha$-rabbit IgG (1:500; ThermoFisher Scientific), respectively.

For the dura staining, CD31 (rabbit- $\alpha$ CD31, 1:200; Abcam, ab28364), Iba1 (rabbit- $\alpha$ Iba1, 1:500; Wako Chemicals, 019-19741), CD68 (rat- $\alpha$ CD68, 1:100; Bio-Rad, MCA1957), Podoplanin (Hamster- $\alpha$ Podoplanin, 1:150; ThermoFisher Scientific, 14538181), and Lyve1 (Rabbit- $\alpha$ Lyve1, 1:100; Abcam, ab14917) was used with the corresponding secondary antibodies, Goat $\alpha$-rabbit IgG (1:500; Abcam), Goat $\alpha$-rat (1:500; Abcam), and Goat $\alpha$-hamster IgG (1:500; ThermoFisher Scientific).

Experimental design and statistical analysis. Statistical analysis was performed using GraphPad Prism v7.0c for Mac OS X. The data are presented as mean \pm SD unless otherwise noted. For comparison of two means, an unpaired Student's $t$ test was used. For statistical analysis of more than two means, we performed ordinary two-way ANOVA with Sidak's multiple-comparisons test and multiple $t$ tests using the HolmSidak method to determine statistical significance, which was ascribed at $p<0.05$. Graphs were generated using GraphPad Prism v7.0c for Mac OS X and Adobe Illustrator CC 2019. For sleep analysis, $48 \mathrm{~h}$ of continuous EEG from five mice was acquired and scored, and analysis of sleepwake time and bouts was performed. All image analysis was done with ImageJ software (NIH). For whole-slice analysis, mean pixel intensity of the whole slice was measured. For surface expression, linear regions-of interest (ROIs) extending inward from the brain surface below the electrodes were selected and the mean pixel intensity profiles were plotted across the regions. The number of lymphatic sprouts, defined as any Proxl-positive vessel sprouting from the lymphatic vasculature at the transverse sinus or superior sagittal sinus, was counted by visual inspection. The length of lymphatic sprouts was measured using the line tool in ImageJ. The number of T cells in the dura was counted by visual inspection of CD3-positive cells in an area of similar size and location between animals. Aquaporin-4 polarization was established by creating rectangular ROIs covering the vessel and surrounding brain tissue (Kress et al., 2014) and then generating plots of the mean pixel intensity perpendicular to the vessels.

\section{Results}

Mice implanted with EEG electrodes exhibited a typical sleepwake architecture

We first assessed whether electrode implantation affected the sleep-wake pattern of mice (Fig. 1A). The mice had electrodes implanted and were placed in the recording setup where they were allowed $3 \mathrm{~d}$ of habituation before video/EEG recordings were started. Age-matched control mice that had not undergone surgery were used as controls. The body weight of mice with EEG electrodes did not differ from the weight of non-implanted control mice (Fig. 1B). To non-invasively assess the sleep-wake pattern, $24 \mathrm{~h}$ video recordings were acquired and periods of immobility was used as a proxy for sleep (Pack et al., 2007). Scoring of immobile periods did not show any significant differences between implanted and non-implanted mice over the $24 \mathrm{~h}$ period (Fig. 1C). The average time spent immobile during the light and dark phases also did not differ between the groups (Fig. 1D), nor did the average distance traveled (Fig. 1E). We next assessed whether the sleep characteristics of EEG implanted mice were similar to those in previously reported studies. Visual inspection of the EEG revealed no sign of seizure activity in any of the mice included in the study (Fig. $1 F$ ). All mice exhibited typical sleepwake behavior with more activity during the dark phase than in the light phase (Fig. $1 G, H$ ). During a $24 \mathrm{~h}$ period, waking accounted for $57.4 \%$, nREM sleep for $35.9 \%$, and REM sleep for 

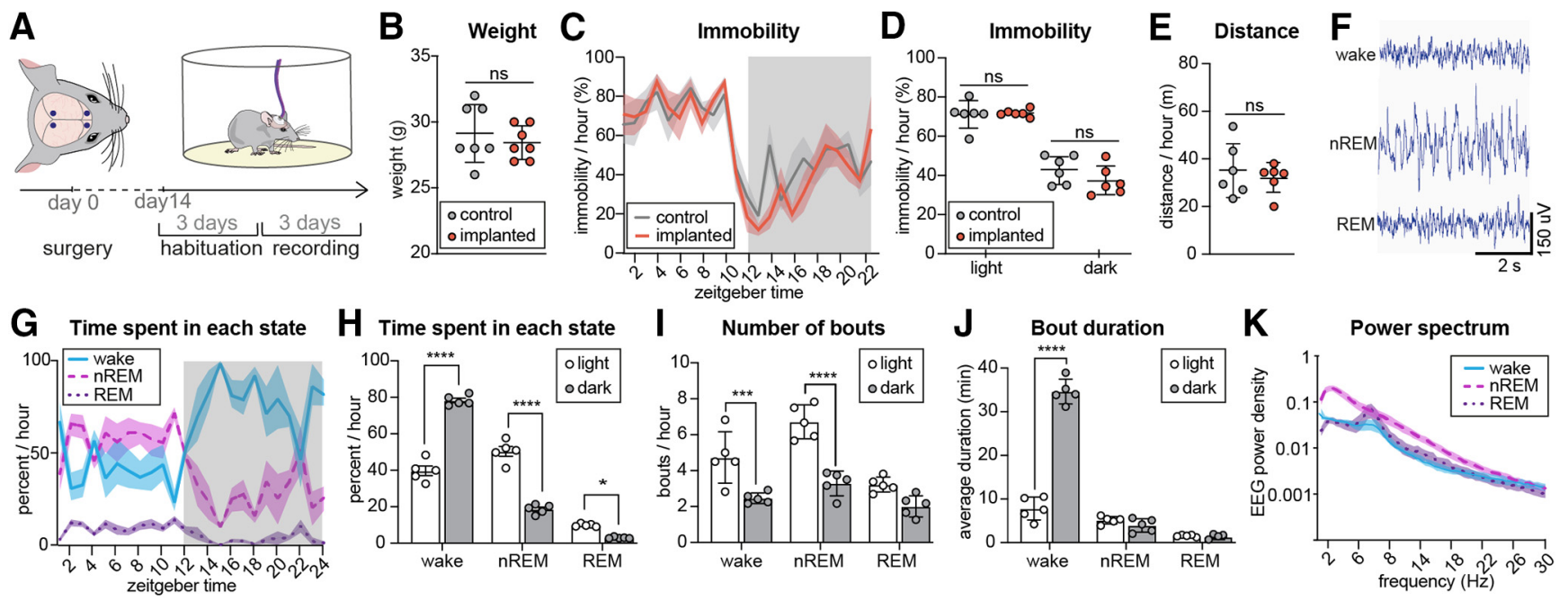

Figure 1. Mice implanted with EEG electrodes exhibited normal sleep architecture. $\boldsymbol{A}$, Experimental time line: $14 \mathrm{~d}$ after electrode implantation, mice were placed in recording chambers and allowed to habituate for $3 \mathrm{~d}$ followed by $3 \mathrm{~d}$ of continuous EEG recording. $\boldsymbol{B}$, The weight of the mice implanted with EEG electrodes did not differ from that of non-implanted control mice (SD, Student's t test, $n=7$ ). C, Mice implanted with electrodes and non-implanted control mice exhibited the same total duration of inactivity over the whole $24 \mathrm{~h}$ light $/$ dark period ( $p=0.26)$ and when every time point was compared (SEM, two-way ANOVA, Sidak's multiple comparison, $n=6$ ), and ( $D$ ) had equal mean durations of immobility in both the light and dark phases (SD, two-way ANOVA, Sidak's multiple comparison, $n=6$ ). $\boldsymbol{E}$, Mice implanted with electrodes and non-implanted control mice traveled a comparable distance over the $24 \mathrm{~h}$ period (SD, Student's $t$ test, $n=6$ ). $\boldsymbol{F}$, Representative images of EEG trace in wakefulness, nREM sleep, and REM sleep. G, Baseline percentage time per hour of waking, nREM, and REM sleep (error bars are SEM). The data shown is derived from $48 \mathrm{~h}$ recordings posed on the same $24 \mathrm{~h}$ timeline. $\boldsymbol{H}$, Mean percentage time spent in waking, nREM and REM sleep. Mice showed a typical light/dark difference with more wakefulness in the dark period and more nREM sleep and REM sleep in the light period (SD, two-way ANOVA, Sidak's multiple comparison, $n=5,{ }^{*} p<0.05,{ }^{* * *} p<0.001,{ }^{* * * *} p<0.0001$ ). I, Average number of wake-, nREM-, and REM bouts per hour during the light and dark phases. Mice had more waking and nREM bouts during the light period (SD, two-way ANOVA, Sidak's multiple comparison, $n=5$, $\left.{ }^{* * *} p<0.001,{ }^{* * *} p<0.0001\right)$. J, Mean bout duration during the light and dark periods. Mice had long bouts of wakefulness during the dark phase (SD, two-way ANOVA, Sidak's multiple comparison, $n=5,{ }^{* * * *} p<0.0001$ ). $K$, EEG power spectra of wake, nREM sleep, and REM sleep from the first $3 \mathrm{~h}$ of the light period (SD; $n=5$ ). ns, non-significant.

$6.7 \%$ of the day. The average number of waking, nREM sleep, and REM sleep bouts (Fig. 1I), and the mean bout durations (1J) also followed the general pattern previously reported in mice (Naylor et al., 2000; Tang and Sanford, 2002; Bellesi et al., 2015; Ho et al., 2016). Also in agreement with existing literature, the mice exhibited a high prevalence of delta power during nREM sleep and a peak in theta power in REM sleep (Huber et al., 2000; Panagiotou et al., 2017; Fig. $1 \mathrm{~K}$ ). From these observations we conclude that the implantation of electrodes was not associated with anomalies in EEG or sleep architecture.

\section{Cranial electrodes induce astrogliosis and microglial activation in underlying brain tissue}

To assess whether the electrodes induce an inflammatory response at the electrode site or underlying brain tissue, mice were implanted with cranial EEG electrodes and the brains collected 3, 20 , or $40 \mathrm{~d}$ later. Investigation of the dura below the electrodes showed intact vasculature as visualized with the endothelial marker CD31, which indicated no mechanical damage to underlying tissue, as might have been caused by drilling or screw insertion (Fig. 2A). Next, we investigated the effect of cranial electrodes on glial scar formation by quantifying the intensity of GFAP, a marker of astrocyte activation, and CD68, a protein expressed by macrophages and activated microglia (Karumbaiah et al., 2013; Lind et al., 2013), in coronal brain sections (Fig. 2B). Cortical tissue in direct contact with electrodes exhibited marked reactive changes. At $3 \mathrm{~d}$ following electrode implantation, we detected at the electrode-dura interface a semicircular area of $\sim 500 \mu \mathrm{m}$ radius containing increased levels of CD68 staining and general absence of defined astrocytic processes. The margins of these pockets of gliosis were surrounded by an outer rim of relatively decreased GFAP signal, which was surrounded by regular tissue displaying distinct astrocytic processes. We next com- pared the intensity of the GFAP and CD68 signals in linear profiles extending inward from the brain surface below the electrodes at 3, 20, and $40 \mathrm{~d}$ post-implantation (Fig. 2C). Surface GFAP expression remained significantly elevated in mice with electrodes across this observation period (Fig. 2D). At $3 \mathrm{~d}$ following electrode implantation, CD68 expression at the electrode site was markedly increased and extended $>700 \mu \mathrm{m}$ below the brain surface (Fig. 2E). At 20 and $40 \mathrm{~d}$ following electrode implantation, CD68 expression was increased in the tissue directly in contact with the electrode $(0-100 \mu \mathrm{m}$ below the surface), whereas CD68 expression in deeper cortex was comparable to that of control animals. In conclusion, our analysis shows that implantation of cranial EEG electrodes is associated with significant reactive responses of both astrocytes and microglia, even in the absence of penetrating tissue injury. These observations are consistent with earlier studies investigating the cortical response to cranial and depth electrodes, which consistently report glial scarring and persistent inflammation around the electrode (Szarowski et al., 2003; Biran et al., 2005; Polikov et al., 2005; Henle et al., 2011; Karumbaiah et al., 2013; Lind et al., 2013; Schendel et al., 2014).

\section{Foreign objects on the cortical surface induce meningeal sprouting from the dural sinuses}

Lymphatic sprouting is a stereotypic response to injury in peripheral tissues (Tammela and Alitalo, 2010). Within the CNS, lymphangiogenesis in response to inflammation has previously been observed only near the cribriform plate (Louveau et al., 2018; Hsu et al., 2019). We set out to assess how the EEG electrodes affected dura mater structures by examining skull-with-dura preparations of brains collected $30 \mathrm{~d}$ after implantation of four electrodes (Fig. 3A). This approach allowed dissection of the entire dura, and thereby a precise in situ localization of the site of electrode 

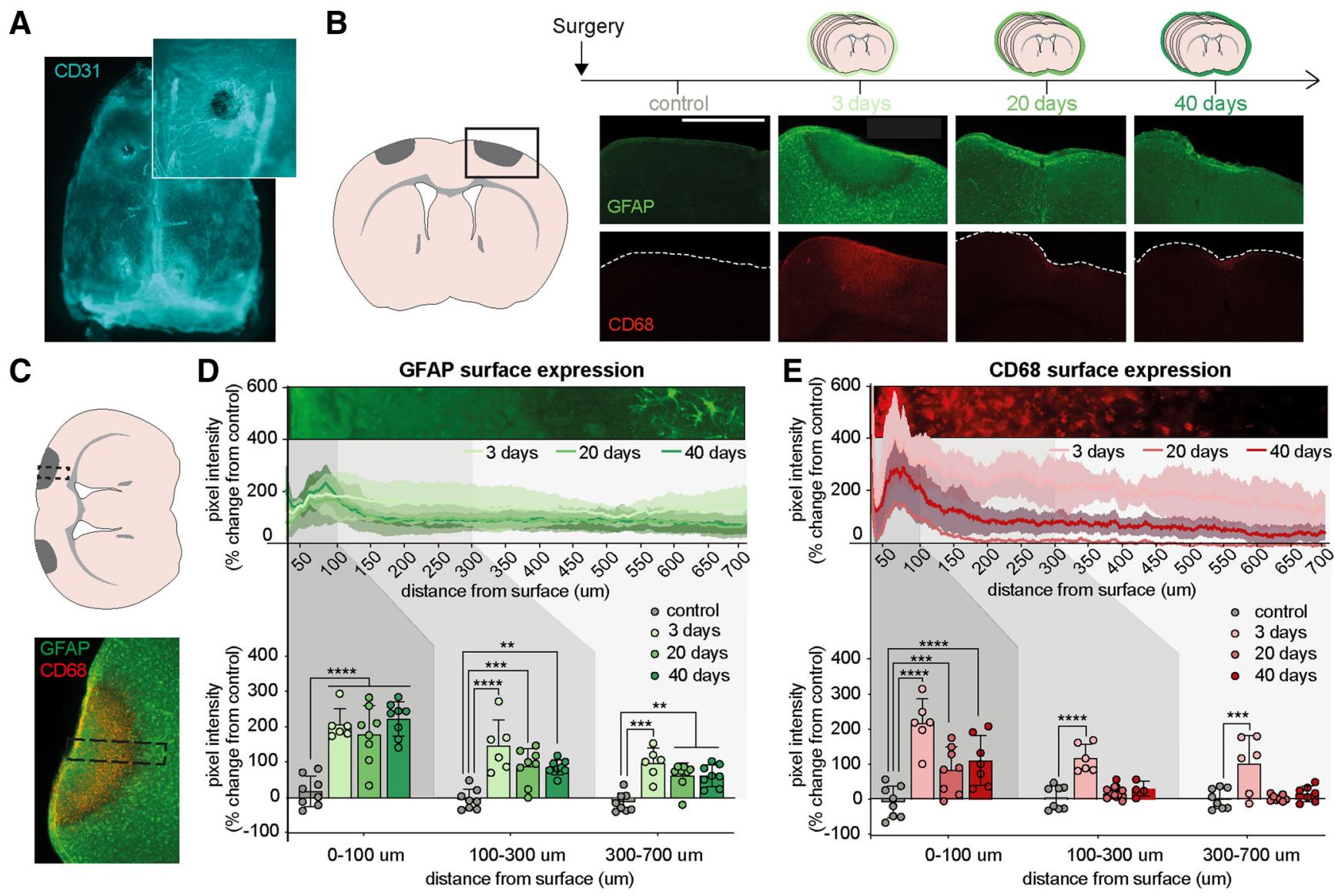

Figure 2. Chronic EEG electrodes induce astrogliosis and glial scarring. $A$, Representative image of skull and dura immunostained for the endothelial marker, $C D 31$. Inset, Higher-magnification image of an electrode burr hole where blood vessels show the continuing presence of intact dura. B, Representative images of brain-electrode interface in coronal slices 3, 20, and $40 \mathrm{~d}$ after implantation. Brains were stained for GFAP (top) and CD68 (bottom). C, GFAP and CD68 immunoreactivity intensities of brain tissue at the brain- electrode interface were analyzed in rectangular ROIs (dotted line) extending inward from the brain surface. D. Mice implanted for 3, 20, and $40 \mathrm{~d}$ showed increased GFAP immunoreactivities under the electrodes at all tissue depths $(0-700 \mu \mathrm{m})$ compared with control mice. Inset, GFAP staining of cortical surface $3 \mathrm{~d}$ after surgery (SD; two-way ANOVA with Dunnett's multiple comparison; ${ }^{* *} p<0.01,{ }^{* * *} p<0.001$, ${ }^{* * * *} p<0.0001$ ). $\boldsymbol{E}$, Three days after surgery, CD68 expression was increased at all tissue depths analyzed. At 20 and $40 \mathrm{~d}$ after surgery, only the tissue $0-100 \mu \mathrm{m}$ under the pial surface showed increased CD68 expression. Inset, CD68 staining of cortical surface $3 \mathrm{~d}$ after surgery (SD; two-way ANOVA with Dunnett's multiple comparison; ${ }^{* * *} p<0.001,{ }^{* * *} p<0.0001$ ).

implantation. When the whole dorsal part of the skull complete with dura was immunolabeled for the endothelial marker CD31, vessels were clearly visible on the entire inner surface of the skull, and burr holes were evident as dark spots (Fig. 3B). Immunolabeling of the skull-dura preparations with Ibal, a marker of macrophages and microglia, and podoplanin, a marker of lymphatic vessels revealed increased Ibal signal at the dura-electrode interface (Fig. $3 C$ ). In the regions in direct contact with the EEG electrodes, immunolabeling revealed podoplanin-positive vessels associated with an increased number of Iba1-positive cells creating knob-like structures with relatively abundant podoplanin expression. This pattern suggested a structural reorganization resembling lymphangiogenesis in response to local inflammation at the dura-electrode interface.

To investigate whether meningeal lymphatic vessels reorganize in response to prolonged presence of EEG electrodes, we examined the dura in Prox-1 EGFP transgenic mice (Choi et al., 2011). Prox1 labels lymphatic vasculature and colocalized with the lymphatic marker Lyve1 (Fig. 3D). In accordance with previous reports, non-implanted control mice exhibited a network of lymphatic vessels running parallel with the transverse sinus and the superior sagittal sinus (Fig. $3 E$ ), and in the bregma region along the middle meningeal artery (Aspelund et al., 2015; Louveau et al., 2015). Furthermore, both in electrode-implanted mice and in control mice, we saw meningeal sprouts arising from lymphatic vessels at the transverse sinus and the superior sagittal sinus while lymphatic vessels surrounding the middle meningeal arteries showed additional organized branching that followed the arterial branches (Fig. $3 F, G$ ). In mice implanted with electrodes, the lymphatic vasculature showed signs of reorganization with lymphatic sprouts stretching toward electrode sites (Fig. $3 H$ ). In high resolution images of whole-mount meninges we quantified the number and length of lymphatic sprouts in the implanted mice. Because the branching of lymphatic vasculature around the middle meningeal artery is difficult to separate from actual sprouts, this analysis was limited to lymphatic sprouting at the superior sagittal sinus and transverse sinus. A quantification of the number of sprouts showed no significant difference between mice implanted with electrodes and non-implanted mice (Fig. $3 I)$, although there was a trend toward more sprouts in mice with electrodes $(p=0.054)$. However, mice with electrodes exhibited significantly longer sprouts compared with non-implanted control mice $(p=0.00098$; Fig. $3 J)$, suggesting that the sprouts extending toward electrodes develop by recruitment from pre-existing, quiescent sprouts.

Our analysis showed that the new sprouts in mice with electrodes mainly originated from the transverse sinus ( $p=0.0318$ ), whereas the superior sagittal sinus had an amount of sprouts 
A

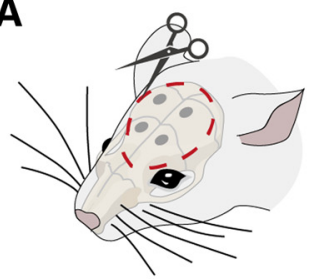

30 days post surgery

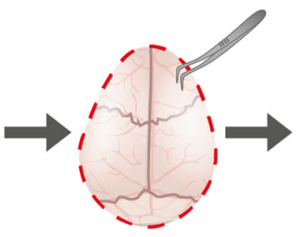

skull + dura

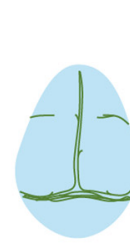

dura
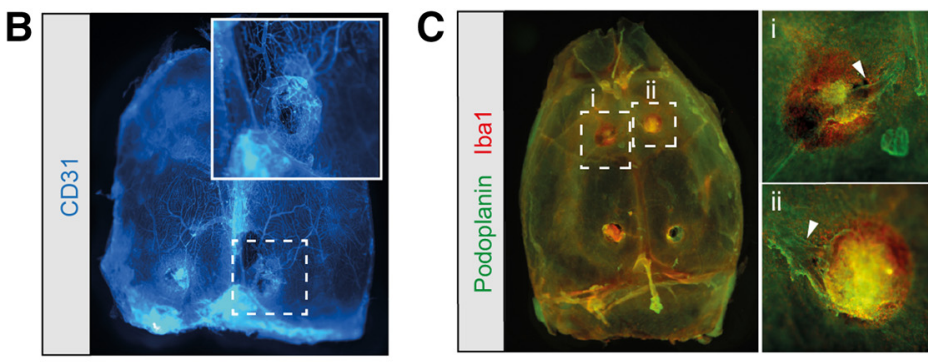
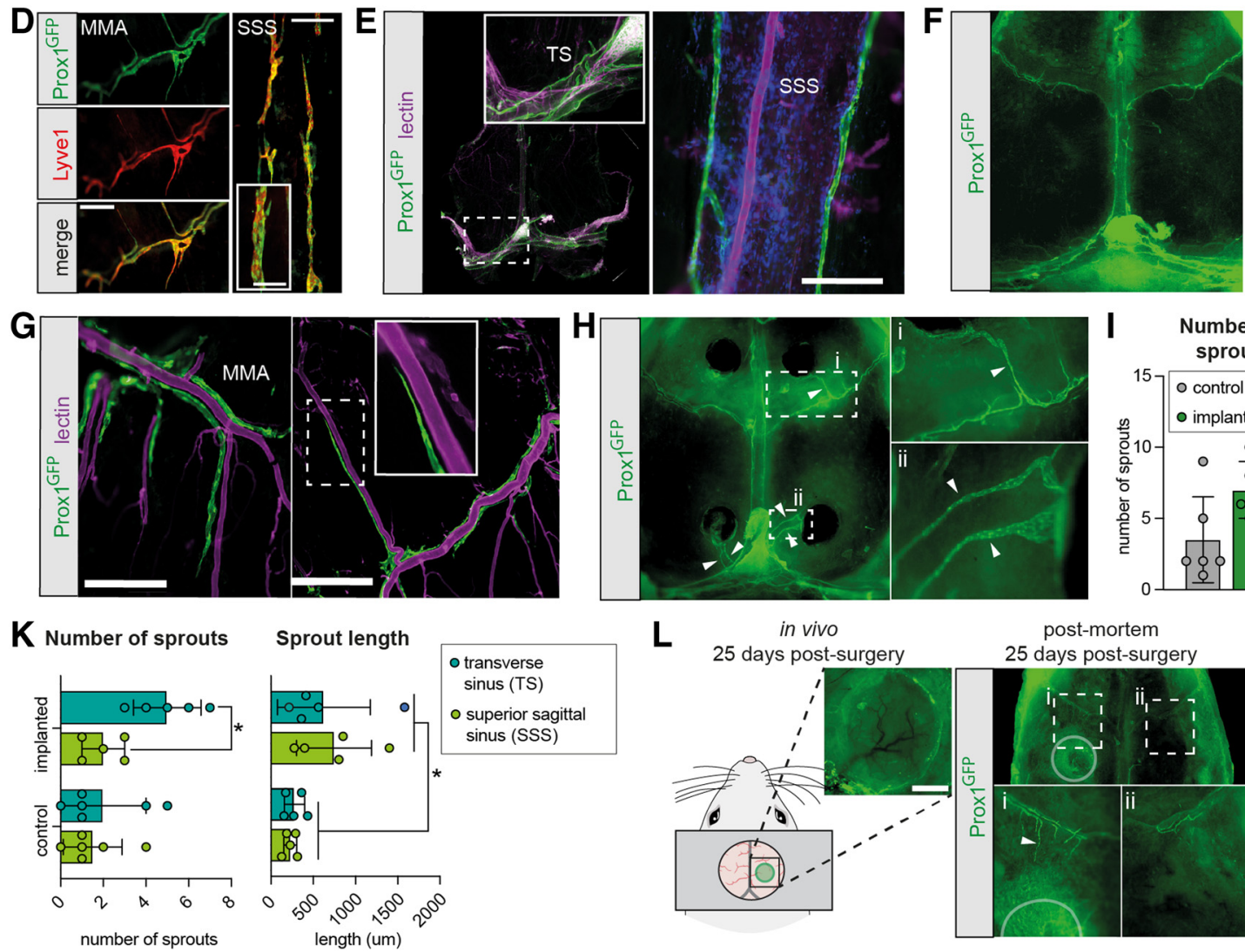

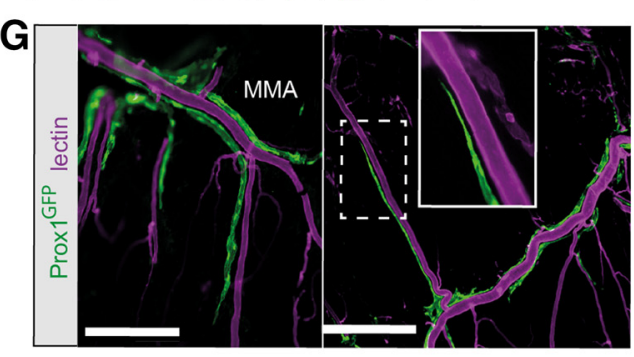

Sprout length

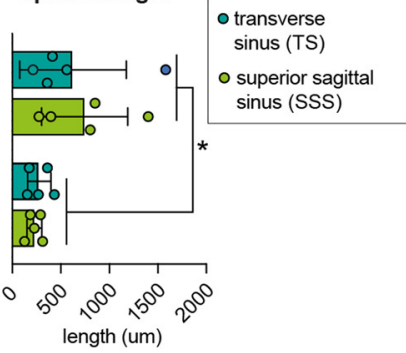

L

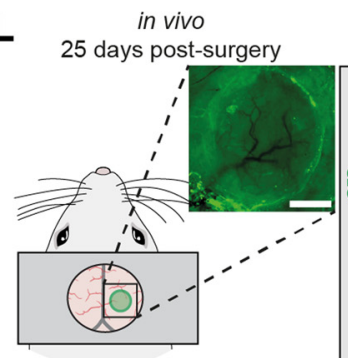

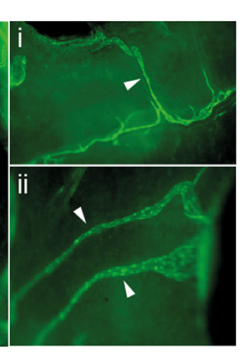

post-mortem 25 days post-surgery

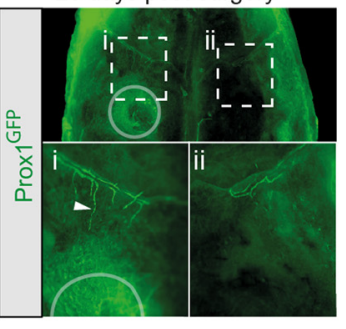

M post-mortem 35 days post-surgery

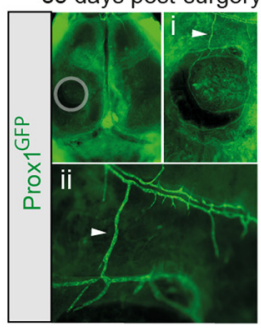

Figure 3. Electrode implantation induces meningeal lymphatic sprouting. $A$, Overview of dura preparations. Thirty days after implantation of electrodes, mice were killed and the top of the skull with the dura in situ was removed. For higher resolution imaging of the dura alone, the dura was dissected carefully from the skull with a pair of tweezers. $\boldsymbol{B}$, Representative image of skull and dura immunostained for the endothelial marker CD31. Inset, A burr hole and surrounding blood vessels. C, Representative image of skull and dura immunostained for the lymphatic marker podoplanin and the microglial marker Iba1. Insets, Higher magnification of increased Iba1 immunoreactivity and reorganization of podoplanin-positive vessels (arrowheads). $\boldsymbol{D}$, Representative image of the middle meningeal artery (MMA) and the superior sagittal sinus (SSS) C0-stained for prox1 GFP (green) and Lyve1 (red). Scale bar, $300 \mu \mathrm{m}$. Inset, Higher magnification of SSS lymphatic vessel. Scale bar, $100 \mu \mathrm{m}$. E, Representative image of meningeal preparation. Lymphatic vessels were mainly observed in the transverse sinus (TS) and the SSS. Scale bar, $200 \mu \mathrm{m}$. Inset, Higher magnification of lymphatic vessels in the TS. $\boldsymbol{F}$, Representative images of skull and dura of control mouse with lymphatic vessels positive for the lymphatic marker Prox 1 (left) and schematic image showing Prox-1-positive vessels (right). Arrowheads point to lymphatic sprouts. Lymphatic vessels were restricted to the SSS, TS, and the MMAs. G, Representative image of meningeal preparation. Lymphatic vessels underlying the bregma region paralleled and branched with the MMA. Scale bars: left, $200 \mu \mathrm{m}$; right, $400 \mu \mathrm{m}$. $\boldsymbol{H}$, Representative images of skull and dura preparation of EEG implanted mouse with Prox1-positive lymphatic vessels showing lymphatic sprouts (arrowheads) reaching toward the electrodes. $I$, Quantification of the number of lymphatic sprouts showed no difference between mice with implants and non-implanted control mice (SD; Student's t test with Holm-Sidak correction; $n=5-6$ ). $J$, Quantification of the lengths of lymphatic sprouts showed significantly longer sprouts in mice with EEG implants than non-implanted control mice (SD; Student's t test with Holm-Sidak correction; $\left.{ }^{* * *} p<0.001 ; n=5-6\right)$. $\boldsymbol{K}$, Quantification of number and length of lymphatic sprouts originating from the TS or the SSS, respectively. Significantly more sprouts initiated from the TS than the SSS in mice with implanted electrodes. Sprouts from mice with electrodes were overall longer than from control mice, but no significant difference in sprout length was found between the SSS and the TS; SD, two-way ANOVA with Sidak's multiple comparison; $\left.{ }^{*} p<0.05 ; n=5-6\right)$. L, Schematic representation of cranial window preparation with inset showing a representative image of the cranial window $25 \mathrm{~d}$ post-surgery (scalebar, $1 \mathrm{~mm}$ ) and representative image of skull-dura preparation 25 d post-surgery. Li, Lymphatic vessels from bregma reaching the cranial window (arrowheads) and (Lii) contralateral hemisphere. $\boldsymbol{M}$, Representative image of skull-dura preparation $35 \mathrm{~d}$ post-surgery. Mi and Mii, Lymphatic vessels from bregma extending to the cranial window (arrowhead) and bone regrowth.

comparable to control mice (Fig. $3 K$ ). However, both the transverse sinus and the superior sagittal sinus had longer sprouts in mice with electrodes compared with non-implanted mice $(p=$ 0.016 ; Fig. $3 K$ ). Thus, these observations show that lymphangiogenesis occurs in mice with cranial electrodes. Moreover, the development of new sprouts occurs predominantly at the lymphatic vasculature in the transverse sinus, while existing sprouts from both the transverse sinus and superior sagittal sinus are significantly elongated in implanted mice compared with control mice.

Last, we sought to investigate the lymphatic response to another commonly used cranially-invasive procedure, i.e., cranial windows, which require chronic implantation of a coverslip on top of the dura. Here, we investigated lymphatic sprouting under 
cranial windows implanted in in Prox-1 mice. In vivo macroscopic imaging from Days 1 to 25 following surgery did not show signs of lymphatic vasculature underneath the cranial window, while postmortem immunohistochemical analysis revealed enhanced Prox-1 signal underneath the cranial window, and new lymphatic vessels arising from the middle meningeal artery and extending toward the cranial window (Fig. $3 \mathrm{~L}$ ). Immunohistochemical analysis of mice $35 \mathrm{~d}$ after surgery revealed some bone regrowth, and presence of lymphatic vessels extending toward the cranial window and, in some cases, surrounding it (Fig. $3 M$ ).

\section{Glymphatic influx is increased in mice implanted with cranial electrodes}

The glymphatic system is defined by its directional flow of CSF from arteries to veins and dependence on aquaporin-4 (AQP4) water channels (Iliff et al., 2012). Previous studies have linked glymphatic clearance to lymphatic drainage by showing that ablation of meningeal lymphatic vessels decreases CSF efflux (Aspelund et al., 2015; Da Mesquita et al., 2018), whereas stimulation of lymphangiogenesis increases the rate of CSF influx (Da Mesquita et al., 2018; Wen et al., 2018). Therefore, we asked whether chronic electrode implantation affects glymphatic fluid transport. To evaluate CSF influx, a tracer (3 KDa fluorescein dextran) was slowly infused into the cisterna magna of awake or ketaminexylazine anesthetized mice $30 \mathrm{~d}$ after electrode implantation (Fig. $4 A$ ). After allowing the CSF tracer to circulate for $30 \mathrm{~min}$, the brains were harvested and immersion fixed in 4\% PFA overnight at $4^{\circ} \mathrm{C}$, before cutting $100-\mu \mathrm{m}$-thick sections spaced evenly across the rostral-caudal axis (Iliff et al., 2012; Kress et al., 2014; von Holstein-Rathlou et al., 2018). Surprisingly, when all brain sections were analyzed for tracer penetration, the ketaminexylazine anesthetized mice implanted with electrodes showed a significantly higher tracer penetration into the brain parenchyma ( $p=0.001$; Fig. $4 B, C)$. Nonetheless, post hoc testing of individual brain slice pairs and average tracer influx for each mouse did not reveal significant differences between the groups $(p=0.11)$. When the experiment was repeated with awake animals, mice implanted with electrodes exhibited a significantly higher tracer penetration into the brain parenchyma than control mice $(p=$ 0.0001 ) across all examined brain sections and significantly higher tracer penetration in the most posterior section (Fig. 4E). Average tracer penetration across all sections was likewise significantly higher for electrode implanted mice ( $p=0.0072$; Fig. $4 F)$.

As tracer influx by visual inspection was most pronounced in the lateral and ventral areas of the brain, we next tested whether the increase in tracer influx was localized to certain brain areas. We compared tracer accumulation in the ventral cerebral cortex, lateral cortex and the medial septum region in mice with and without previously implanted EEG electrodes (Fig. 4H). All groups exhibited the highest tracer influx into the medial septum region. Awake mice with EEG electrodes had significantly higher tracer penetration into the medial septum region compared with non-implanted controls $(p=0.0009)$ but no difference for the other regions analyzed (Fig. 4I). Mice anesthetized with ketamine-xylazine did not exhibit significant differences in tracer penetration between electrode-implanted mice and controls in either of the regions analyzed.

To examine whether chronic inflammation was present in meninges from mice with electrodes, meningeal preparations from the same mice were immunostained for CD3 ( $\mathrm{T}$ cells; Fig. $4 J)$. As previously reported, $\mathrm{T}$ cells were present in both the dura and the superior sagittal sinus (Louveau et al., 2015), but we observed no significant difference in the numbers of T-cells when comparing implanted and non-implanted mice (Fig. 4K). Finally, we assessed the effect of electrode implantation on expression and polarization of AQP4 (Fig. 4L). No significant difference in AQP4 expression between mice with electrodes and nonimplanted control mice was noted (Fig. 4M), nor did the AQP4 polarization index differ between the two groups $(4 N)$. Thus, our analysis suggests that the increased glymphatic influx occurred in the absence of clear signs of inflammatory changes in the dura or loss of APQ4 polarization.

\section{Discussion}

We here analyzed the effect of chronically implanted EEG electrodes on glymphatic function and structural remodeling of the dura. Our key observation is that meningeal lymphatic vessels of mice $30 \mathrm{~d}$ after the implantation of electrodes exhibit lymphangiogenesis in the vicinity of the EEG electrodes (Fig. $3 H$ ), along with widespread reactive gliosis (Fig. 2), and furthermore that CSF influx was significantly increased in these mice (Fig. 4). The pathological responses to the EEG electrode implantation were not a consequence of poor technique resulting in brain injury; the mice implanted with EEG electrodes exhibited a normal sleepwake pattern and no abnormal brain activity such as seizures (Fig. 1). Also, immunohistochemical staining of meninges with the endothelial marker CD31 showed presence of intact blood vessels below the electrodes, further demonstrating that the dura was not penetrated by the electrodes (Fig. 2A).

We observed a chronic increase in GFAP and CD68 immunoreactivities at the brain-electrode interface; a classical sign of glial scarring. This finding of astroglia and microglia reactivity is in general agreement with previous studies noting thickened and fibrotic tissue below the electrode site (Henle et al., 2011; Karumbaiah et al., 2013). Similar findings have previously been reported. One such study found increased GFAP immunoreactivity in a large cortical area surrounding an array of EEG electrodes 1 week after implantation, although elevated GFAP staining 6 weeks after implantation was mainly confined to a region 50-100 $\mu \mathrm{m}$ around the electrodes (Szarowski et al., 2003). Another study reported increased GFAP immunoreactivity in response to a chronically implanted electrode 1 and 3 weeks post-surgery, but lower and more circumscribed GFAP signal at 6 weeks postsurgery (Xie et al., 2014).

In whole-mount preparations of skull and dura from mice, we observed extensive lymphangiogenesis in the meningeal lymphatic vasculature lying close to the electrodes (Fig. 3). In particular, new lymphatic vessels had sprouted from the dural sinuses and from lymphatic vessels at the bregma region, with most sprouts originating from the transverse sinus. It has previously been reported in a mouse model of multiple sclerosis that lymphangiogenesis occurred near the cribriform plate, but was not evident in the meninges (Louveau et al., 2018). Nevertheless, present observations show that meningeal lymphangiogenesis can occur in response to implantation of chronic EEG electrodes. We further sought to investigate the lymphatic response to another representative and commonly used cranially-invasive research procedures. To this end, we installed cranial windows in mice and again noted formation of lymphatic sprouts extending toward the cranial window (Fig. $3 L$ ). Thus, the effects of lymphatic reorganization must also be considered in imaging experiments based on preparations of chronic cranial windows. We conclude that meningeal lymphangiogenesis occurs in response to both procedures, and that this phenomenon likely is part of the reactive changes noted in the meninges and the underlying cortical tissue. 
A

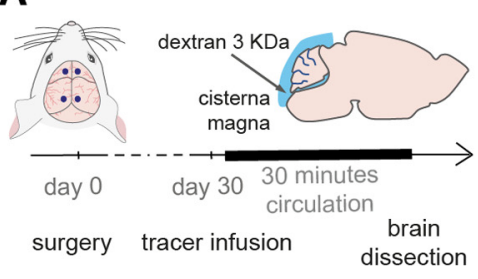

B

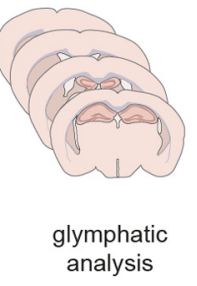

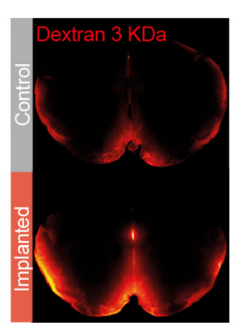

C

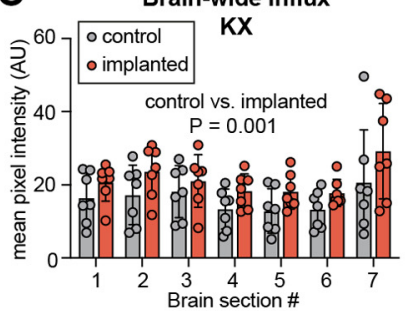

D Brain-wide influx

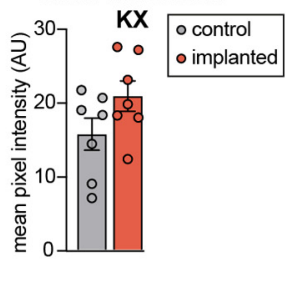

E

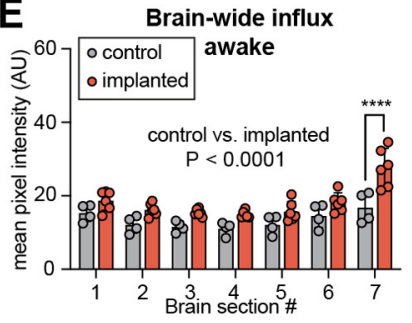

I

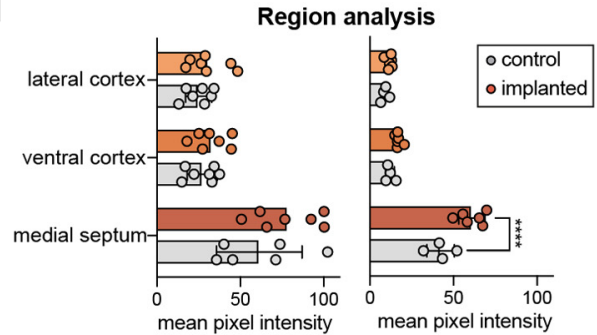

G

F Brain-wide influx

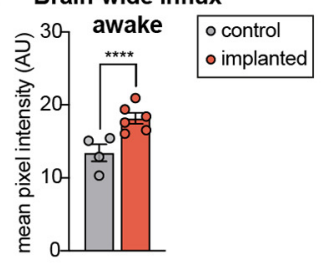

H

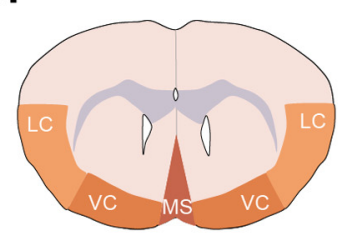

J
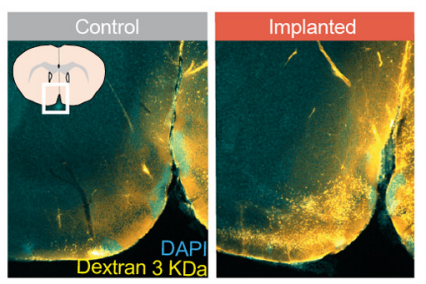

superior sagittal sinus
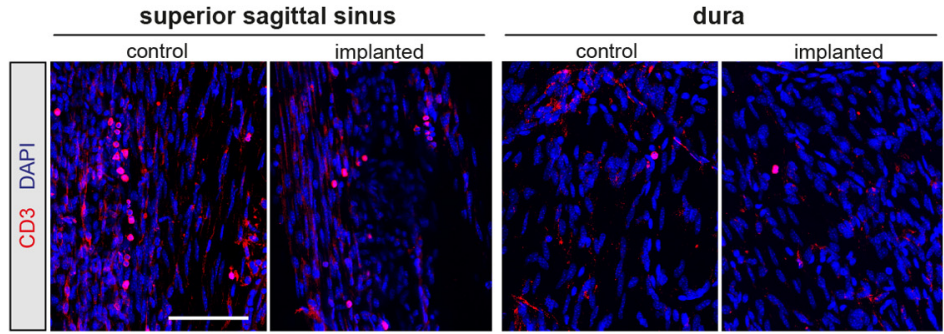

M

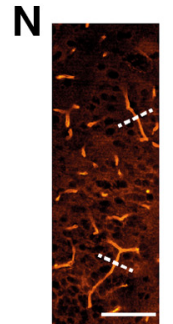

AQP4 intensity projection

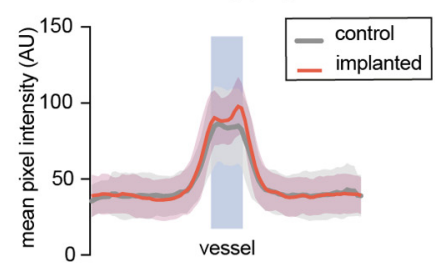

Figure 4. Glymphatic CSF tracer influx is enhanced in mice with chronic EGG electrodes. $\boldsymbol{A}$, Experimental timeline: mice were examined $30 \mathrm{~d}$ after implantation of cranial electrodes. A CSF tracer (fluorescein dextran $3 \mathrm{KDa}$ ) was infused via a cannula inserted into the cisterna magna of awake mice or mice under ketamine-xylazine anesthesia. Following 30 min of tracer circulation, the brain was dissected out, cut on a vibratome, and six brain sections spaced evenly across the anterior-posterior axis were analyzed for tracer influx. $\boldsymbol{B}$, Representative images showing increased tracer penetration in mice implanted with electrodes compared with non-implanted control mice (both groups under ketamine-xylazine anesthesia). C, Quantification of tracer influx in mice under ketamine-xylazine anesthesia measured by the mean pixel intensity for each brain section showed an overall significant difference ( $p=0.001)$ between implanted and non-implanted mice. Post hoc testing did not show significant differences between individual brain sections (error bars are SD; two-way ANOVA with Sidak's multiple comparison; $n=7$ ). $D$, Average tracer influx of all brain sections was not significantly different between implanted mice and control mice ( $p=0.111$; Student's $t$ test). $\boldsymbol{E}$, Quantification of tracer influx for each brain section in awake mice showed an overall significant difference ( $p=0.0001$ ) between implanted and non-implanted mice and post hoc test showed a significant difference between the groups for most posterior brain section (error bars are SD; tw0-way ANOVA with Sidak's correction for multiple comparison; $n=4-6$; $\left.{ }^{* * * *} p<0.0001\right)$. $\boldsymbol{F}$, Average tracer influx of all brain sections showed a significant difference ( $p=0.0072$ ) between implanted mice and control mice (Student's t test; $n=4-6$; ${ }^{* * *} p<0.0001$ ). G, Representative image of tracer penetration in electrode-implanted mouse and non-implanted control mouse (both injected while awake) showing enhanced tracer penetration in the medial septum region and central cortex. $\boldsymbol{H}$, Regional analysis of tracer penetration was performed in the lateral cortex (LC), ventral cortex (VC), and medial septum (MS). $I$, The greatest tracer penetration occurred in the medial septum region for all groups. Regional analysis of the medial septum region showed a significant difference between mice with electrodes and non-implanted control mice in the awake group, but no significant difference for the other regions analyzed or for mice under ketamine-xylazine anesthesia (SD, two-way ANOVA with Sidak's correction for multiple comparison; $n=4-7$; ${ }^{* * * *} p<0.0001$ ). J, Representative confocal images of CD3-expressing cells in whole-mount meninges. Scale bar, $100 \mu \mathrm{m}$. $\boldsymbol{K}$, Quantification of the number of T cells in the superior sagittal sinus and in dura showed no significant difference between implanted and non-implanted animals (SD, two-way ANOVA with Sidak's multiple comparison; $n=5-6$ ). $\boldsymbol{L}$, Representative image of brain slice stained for AQP4. Scale bar, $200 \mu m$. $M$, Quantification of AQP4 expression in the brain area depicted in $L$ measured as mean pixel intensity did not show a difference between mice with EEG electrodes and non-implanted control mice (SD, Student's $t$ test with Holm-Sidak correction; $n=7) . N, A Q P 4$ immunofluorescence was measured in linear regions of interest (dashed lines) across blood vessels in the same brain area and did not show significant differences between the groups (SD; multiple $t$ tests with Holm-Sidak correction; $n=7$ ). Scale bar, $200 \mu \mathrm{m}$. AU, Arbitrary units; KX, ketamine-xylazine.

In groups of mice implanted with electrodes and nonimplanted control mice, we injected a $3 \mathrm{KDa}$ fluorescent tracer into the cisterna magna and followed its distribution in brain as a marker of glymphatic influx in anesthetized and awake mice $30 \mathrm{~d}$ after implantation (Fig. 4). Glymphatic CSF influx was increased in both awake and anesthetized mice. These observations support previous observations suggesting that glymphatic clearance is regulated by lymphatic drainage
(Aspelund et al., 2015; Louveau et al., 2015, 2018; Ma et al., 2019). At the same time, this finding was surprising because astrogliosis and microgliosis in aging or following traumatic brain injury and stroke were previously reported to impair glymphatic function (Gaberel et al., 2014; Iliff et al., 2014; Kress et al., 2014; Plog and Nedergaard, 2018; Rasmussen et al., 2018). Our observations suggest that reactive gliosis is not necessarily linked to reduced glymphatic activity. 
Electrode-implanted mice both in the awake and the anesthetized groups showed higher tracer penetration than did nonimplanted control mice, when data from all brain sections were included in the analysis. However, electrode-implanted mice in the anesthesia group did not exhibit significantly higher tracer influx when brain sections from each mouse were averaged and compared between the groups. This discrepancy might be because of the increased variability of tracer signal within the anesthesia-group dataset (coefficient of variation $=26-37 \%$ for the anesthetized group and $10-18 \%$ for the awake group). This difference in variability could be due to variable depth of anesthesia at the time of tracer infusion, despite efforts to administer dosing of anesthesia based on the weight of the individual mice. Alpha $_{2}$-adrenergic agonists such as xylazine increase glymphatic influx by increasing slow-wave activity and reducing cardiac pulsatility (Hablitz et al., 2019), implying that small differences in the depth of anesthesia might therefore result in the present variability in glymphatic activity. Of note, mice in the anesthesia group had tracer infused immediately following surgery, whereas mice in the awake group were allowed to recover for $2 \mathrm{~d}$. This design difference may also account for the higher variance in the anesthesia group.

We speculate that the increase in glymphatic function in mice with electrode implantation results from increased meningeal lymphangiogenesis. Two prior studies have shown that brain glymphatic clearance is boosted in response to meningeal lymphangiogenesis. In one study, adeno-associated virus-delivered vascular endothelial growth factor (VEGF) was used to treat two different transgenic mouse models of Alzheimer's disease, i.e., the $\mathrm{J} 20$ transgenic mice and 5xFAD transgenic mouse, as well as aged wild-type mice, aiming to increase lymphangiogenesis as a therapeutic intervention. Following VEGF treatment, mice showed significantly larger meningeal lymphatic vessels, and interestingly, also significantly higher rate of glymphatic tracer influx into the brain parenchyma, as well as enhanced tracer drainage to deep cervical lymph nodes (Da Mesquita et al., 2018). In another study, meningeal lymphangiogenesis was induced in APP/PS1 transgenic mice by treatment with recombinant human VEGF. Upon this treatment, the authors observed decreased amyloid- $\beta$ levels in CSF and brain, and increased amyloid- $\beta$ clearance to the cervical lymph nodes compared with that in untreated mice (Wen et al., 2018).

On the other hand, a recent study by Ahn et al. (2019) showed that meningeal lymphatic vessels in the basal parts of the skull, but not in dorsal meninges, are mainly responsible for CSF efflux. In other words, the dorsal lymphangiogenesis monitored in this study might not be a direct cause for the increased glymphatic influx. We did not investigate whether electrode implantation also resulted in distal lymphangiogenesis at other locations, such as the basal lymphatic vasculature, where lymphangiogenesis was previously shown to occur in a mouse model of multiple sclerosis (Louveau et al., 2018; Hsu et al., 2019). In this study, we observed the largest influx of tracer in the more ventral regions of the brain, and lesser influx in the dorsal part lying closest to the electrodes, which could imply that lymphatic/vascular changes also occur in the ventral brain regions. Future studies should investigate whether cortical implants also induce distal lymphangiogenesis in the basal lymphatic vasculature and in draining lymph nodes.

Our method for EEG electrode implantation follows standard laboratory procedures for EEG electrode placement (Mang and Franken, 2012; Fernandez et al., 2017). However, in any study using chronically implanted electrodes, it is imperative to consider possible side-effects the surgery and/or the electrodes them- selves might have on experimental endpoints. EEG is the gold standard for investigating sleep, but factors like inflammation caused by EEG electrodes could provoke changes in the sleep pattern (Ranjbaran et al., 2007). One study reported that EEG electrode implantation increased seizure susceptibility in mice. Furthermore, EEG implanted mice exhibited significantly more microglial activation after experiencing seizures than did their sham-operated controls (Balzekas et al., 2016). We suggest that immunohistochemical analysis of the brain should always be conducted after such experiments to exclude animals exhibiting significant inflammation or tissue damage of the brain or dura.

Overall, this study provides the, to our knowledge, first demonstration that common surgical procedures such as implantation of EEG electrodes and chronic cranial window implantation induce meningeal lymphangiogenesis and increased glymphatic CSF circulation. Furthermore, we present direct evidence that dispersion of a CSF tracer in the brain parenchyma, i.e., glymphatic flow, is enhanced following chronic cranial electrode implantation. These findings shed new light on how the brain reacts to focal neuroinflammation and emphasizes how standard invasive procedures can have both local and global long-term effects on brain homeostasis.

\section{References}

Absinta M, Ha SK, Nair G, Sati P, Luciano NJ, Palisoc M, Louveau A, Zaghloul KA, Pittaluga S, Kipnis J, Reich DS (2017) Human and nonhuman primate meninges harbor lymphatic vessels that can be visualized noninvasively by MRI. eLife 6:e29738.

Ahn JH, Cho H, Kim JH, Kim SH, Ham JS, Park I, Suh SH, Hong SP, Song JH, Hong YK, Jeong Y, Park SH, Koh GY (2019) Meningeal lymphatic vessels at the skull base drain cerebrospinal fluid. Nature 572:62-66.

Antila S, Karaman S, Nurmi H, Airavaara M, Voutilainen MH, Mathivet T, Chilov D, Li Z, Koppinen T, Park JH, Fang S, Aspelund A, Saarma M, Eichmann A, Thomas JL, Alitalo K (2017) Development and plasticity of meningeal lymphatic vessels. J Exp Med 214:3645-3667.

Aspelund A, Antila S, Proulx ST, Karlsen TV, Karaman S, Detmar M, Wiig H, Alitalo K (2015) A dural lymphatic vascular system that drains brain interstitial fluid and macromolecules. J Exp Med 212:991-999.

Balzekas I, Hernandez J, White J, Koh S (2016) Confounding effect of EEG implantation surgery: inadequacy of surgical control in a two hit model of temporal lobe epilepsy. Neurosci Lett 622:30-36.

Bellesi M, de Vivo L, Tononi G, Cirelli C (2015) Effects of sleep and wake on astrocytes: clues from molecular and ultrastructural studies. BMC Biol 13:66.

Benveniste H, Diemer NH (1987) Cellular reactions to implantation of a microdialysis tube in the rat hippocampus. Acta Neuropathol 74:234238.

Benveniste H, Lee H, Ding F, Sun Q, Al-Bizri E, Makaryus R, Probst S, Nedergaard M, Stein EA, Lu H (2017) Anesthesia with dexmedetomidine and low-dose isoflurane increases solute transport via the glymphatic pathway in rat brain when compared with high-dose isoflurane. Anesthesiology 127:976-988.

Biran R, Martin DC, Tresco PA (2005) Neuronal cell loss accompanies the brain tissue response to chronically implanted silicon microelectrode arrays. Exp Neurol 195:115-126.

Cai R, Pan C, Ghasemigharagoz A, Todorov MI, Förstera B, Zhao S, Bhatia HS, Parra-Damas A, Mrowka L, Theodorou D, Rempfler M, Xavier ALR, Kress BT, Benakis C, Steinke H, Liebscher S, Bechmann I, Liesz A, Menze B, Kerschensteiner M, et al. (2019) Panoptic imaging of transparent mice reveals whole-body neuronal projections and skull-meninges connections. Nat Neurosci 22:317-327.

Choi I, Chung HK, Ramu S, Lee HN, Kim KE, Lee S, Yoo J, Choi D, Lee YS, Aguilar B, Hong YK (2011) Visualization of lymphatic vessels by Prox1promoter directed GFP reporter in a bacterial artificial chromosomebased transgenic mouse. Blood 117:362-365.

Da Mesquita S, Louveau A, Vaccari A, Smirnov I, Cornelison RC, Kingsmore KM, Contarino C, Onengut-Gumuscu S, Farber E, Raper D, Viar KE, Powell RD, Baker W, Dabhi N, Bai R, Cao R, Hu S, Rich SS, Munson JM, 
Lopes MB, et al. (2018) Functional aspects of meningeal lymphatics in ageing and Alzheimer's disease. Nature 560:185-191.

Fernandez LMJ, Lecci S, Cardis R, Vantomme G, Béard E, Lüthi A (2017) Quantifying infra-slow dynamics of spectral power and heart rate in sleeping mice. J Vis Exp 126:e55863.

Fultz NE, Bonmassar G, Setsompop K, Stickgold RA, Rosen BR, Polimeni JR, Lewis LD (2019) Coupled electrophysiological, hemodynamic, and cerebrospinal fluid oscillations in human sleep. Science 366:628-631.

Gaberel T, Gakuba C, Goulay R, Martinez De Lizarrondo SM, Hanouz JL, Emery E, Touze E, Vivien D, Gauberti M (2014) Impaired glymphatic perfusion after strokes revealed by contrast-enhanced MRI. Stroke 45:3092-3096.

Goldey GJ, Roumis DK, Glickfeld LL, Kerlin AM, Reid RC, Bonin V, Schafer DP, Andermann ML (2014) Removable cranial windows for long-term imaging in awake mice. Nat Protoc 9:2515-2538.

Goss-Varley M, Shoffstall AJ, Dona KR, McMahon JA, Lindner SC, Ereifej ES, Capadona JR (2018) Rodent behavioral testing to assess functional deficits caused by microelectrode implantation in the rat motor cortex. J Vis Exp 138:e57829.

Hablitz LM, Vinitsky HS, Sun Q, Stæger FF, Sigurdsson B, Mortensen KN, Lilius TO, Nedergaard M (2019) Increased glymphatic influx is correlated with high EEG delta power and low heart rate in mice under anesthesia. Sci Adv 5:eaav5447.

Henle C, Raab M, Cordeiro JG, Doostkam S, Schulze-Bonhage A, Stieglitz T, Rickert J (2011) First long term in vivo study on subdurally implanted micro-ECoG electrodes, manufactured with a novel laser technology. Biomed Microdevices 13:59-68.

Ho JM, Barf RP, Opp MR (2016) Effects of sleep disruption and high fat intake on glucose metabolism in mice. Psychoneuroendocrinology 68: $47-56$.

Hsu M, Rayasam A, Kijak JA, Choi YH, Harding JS, Marcus SA, Karpus WJ, Sandor M, Fabry Z (2019) Neuroinflammation-induced lymphangiogenesis near the cribriform plate contributes to drainage of CNS-derived antigens and immune cells. Nat Commun 10:229.

Huber R, Deboer T, Tobler I (2000) Topography of EEG dynamics after sleep deprivation in mice. J Neurophysiol 84:1888-1893.

Iliff JJ, Wang M, Liao Y, Plogg BA, Peng W, Gundersen GA, Benveniste H, Vates GE, Deane R, Goldman SA, Nagelhus EA, Nedergaard M (2012) A paravascular pathway facilitates CSF flow through the brain parenchyma and the clearance of interstitial solutes, including amyloid. Sci Transl Med 4:147ra111.

Iliff JJ, Chen MJ, Plog BA, Zeppenfeld DM, Soltero M, Yang L, Singh I, Deane $R$, Nedergaard M (2014) Impairment of glymphatic pathway function promotes tau pathology after traumatic brain injury. J Neurosci 34:16180-16193.

Ji RC (2007) Lymphatic endothelial cells, inflammatory lymphangiogenesis, and prospective players. Curr Med Chem 14:2359-2368.

Karumbaiah L, Saxena T, Carlson D, Patil K, Patkar R, Gaupp EA, Betancur M, Stanley GB, Carin L, Bellamkonda RV (2013) Relationship between intracortical electrode design and chronic recording function. Biomaterials 34:8061-8074.

Kress BT, Iliff JJ, Xia M, Wang M, Wei HS, Zeppenfeld D, Xie L, Kang H, Xu Q, Liew JA, Plog BA, Ding F, Deane R, Nedergaard M (2014) Impairment of paravascular clearance pathways in the aging brain. Ann Neurol 76:845-861.

Landis DM (1994) The early reactions of non-neuronal cells to brain injury. Annu Rev Neurosci 17:133-151.

Libourel PA, Corneyllie A, Luppi PH, Chouvet G, Gervasoni D (2015) Unsupervised online classifier in sleep scoring for sleep deprivation studies. Sleep 38:815-828.

Lind G, Linsmeier CE, Schouenborg J (2013) The density difference between tissue and neural probes is a key factor for glial scarring. Sci Rep 3:2942.

Louveau A,Herz J, Alme MN, Salvador AF, Dong MQ, Viar KE, Herod SG, Knopp J, Setliff JC, Lupi AL, Da Mesquita S, Frost EL, Gaultier A, Harris TH, Cao R, Hu S, Lukens JR, Smirnov I, Overall CC, Oliver G, et al. (2018) CNS lymphatic drainage and neuroinflammation are regulated by meningeal lymphatic vasculature. Nat Neurosci 21:1380-1391.

Louveau A, Smirnov I, Keyes TJ, Eccles JD, Rouhani SJ, Peske JD, Derecki NC, Castle D, Mandell JW, Lee KS, Harris TH, Kipnis J (2015) Structural and functional features of central nervous system lymphatic vessels. Nature 523:337-341.
Ma Q, Ries M, Decker Y, Müller A, Riner C, Bücker A, Fassbender K, Detmar M, Proulx ST (2019) Rapid lymphatic efflux limits cerebrospinal fluid flow to the brain. Acta Neuropathol 137:151-165.

Mang GM, Franken P (2012) Sleep and EEG phenotyping in mice. In: Current protocols in mouse biology, pp 55-74. Hoboken, NJ: Wiley.

Naylor E, Bergmann BM, Krauski K, Zee PC, Takahashi JS, Vitaterna MH, Turek FW (2000) The circadian clock mutation alters sleep homeostasis in the mouse. J Neurosci 20:8138-8143.

Pack AI, Galante RJ, Maislin G, Cater J, Metaxas D, Lu S, Zhang L, Von Smith R Von, Kay T, Lian J, Svenson K, Peters LL (2007) Novel method for high-throughput phenotyping of sleep in mice. Physiol Genomics 28:232-238.

Panagiotou M, Vyazovskiy VV, Meijer JH, Deboer T (2017) Differences in electroencephalographic non-rapid-eye movement sleep slow-wave characteristics between young and old mice. Sci Rep 7:43656.

Plog BA, Nedergaard M (2018) The glymphatic system in central nervous system health and disease: past, present, and future. Annu Rev Pathol 13:379-394.

Polikov VS, Tresco PA, Reichert WM (2005) Response of brain tissue to chronically implanted neural electrodes. J Neurosci Methods 148:1-18.

Ranjbaran Z, Keefer L, Stepanski E, Farhadi A, Keshavarzian A (2007) The relevance of sleep abnormalities to chronic inflammatory conditions. Inflamm Res 56:51-57.

Rasmussen MK, Mestre H, Nedergaard M (2018) The glymphatic pathway in neurological disorders. Lancet Neurol 17:1016-1024.

Roman A, Meftah S, Arthaud S, Luppi PH, Peyron C (2018) The inappropriate occurrence of rapid eye movement sleep in narcolepsy is not due to a defect in homeostatic regulation of rapid eye movement sleep. Sleep 41:zsy046.

Rosier M, Le Barillier L, Meunier D, El Yacoubi M, Malleret G, Salin PA (2018) Post-learning paradoxical sleep deprivation impairs reorganization of limbic and cortical networks associated with consolidation of remote contextual fear memory in mice. Sleep 41:zsy188.

Saijo K, Glass CK (2011) Microglial cell origin and phenotypes in health and disease. Nat Rev Immunol 11:775-787.

Sawyer AJ, Kyriakides TR (2013) Nanoparticle-based evaluation of bloodbrain barrier leakage during the foreign body response. J Neural Eng 10:016013.

Schendel AA, Nonte MW, Vokoun C, Richner TJ, Brodnick SK, Atry F, Frye S, Bostrom P, Pashaie R, Thongpang S, Eliceiri KW, Williams JC (2014) The effect of micro-ECoG substrate footprint on the meningeal tissue response. J Neural Eng 11:046011.

Silasi G, Boyd JD, LeDue J, Murphy TH (2013) Improved methods for chronic light-based motor mapping in mice: automated movement tracking with accelerometers, and chronic EEG recording in a bilateral thinskull preparation. Front Neural Circuits 7:123.

Szarowski DH, Andersen MD, Retterer S, Spence AJ, Isaacson M, Craighead HG, Turner JN, Shain W (2003) Brain responses to micro-machined silicon devices. Brain Res 983:23-35.

Tammela T, Alitalo K (2010) Lymphangiogenesis: molecular mechanisms and future promise. Cell 140:460-476.

Tang X, Sanford LD (2002) Telemetric recording of sleep and home cage activity in mice. Sleep 25:691-699.

Turner JN, Shain W, Szarowski DH, Andersen M, Martins S, Isaacson M, Craighead H (1999) Cerebral astrocyte response to micromachined silicon implants. Exp Neurol 156:33-49.

Visanji NP, Lang AE, Munoz DG (2018) Lymphatic vasculature in human dural superior sagittal sinus: implications for neurodegenerative proteinopathies. Neurosci Lett 665:18-21.

von Holstein-Rathlou S, Petersen NC, Nedergaard M (2018) Voluntary running enhances glymphatic influx in awake behaving, young mice. Neurosci Lett 662:253-258

Wen YR, Yang JH, Wang X, Yao ZB (2018) Induced dural lymphangiogenesis facilities soluble amyloid-beta clearance from brain in a transgenic mouse model of Alzheimer's disease. Neural Regen Res 13:709-716.

Xie L, Kang H, Xu Q, Chen MJ, Liao Y, Thiyagarajan M, O’Donnell J, Christensen DJ, Nicholson C, Iliff JJ, Takano T, Deane R, Nedergaard M (2013) Sleep drives metabolite clearance from the adult brain. Science 342:373-377.

Xie Y, Martini N, Hassler C, Kirch RD, Stieglitz T, Seifert A, Hofmann UG (2014) In vivo monitoring of glial scar proliferation on chronically implanted neural electrodes by fiber optical coherence tomography. Front Neuroeng 7:34. 